\title{
SRSF2 Mutations in Uveal Melanoma: A Preference for In-Frame Deletions?
}

\author{
Natasha M. van Poppelen 1,2 ${ }^{\mathbb{D}}$, Wojtek Drabarek ${ }^{1,2}$, Kyra N. Smit ${ }^{1,2}$, Jolanda Vaarwater ${ }^{1,2}$, \\ Tom Brands ${ }^{1,2}$, Dion Paridaens ${ }^{1,3}$, Emine Kiliç ${ }^{1,+}$ and Annelies de Klein ${ }^{2, *, \dagger}$ \\ 1 Department of Ophthalmology, Erasmus University Medical Center, 3015 GD Rotterdam, The Netherlands \\ 2 Department of Clinical Genetics, Erasmus University Medical Center, 3015 GD Rotterdam, The Netherlands \\ 3 The Rotterdam Eye Hospital, 3011 BH Rotterdam, The Netherlands \\ * Correspondence: a.deklein@erasmusmc.nl; Tel.: +31-10-704-4153 \\ + Co-senior author.
}

Received: 30 May 2019; Accepted: 15 August 2019; Published: 17 August 2019

\begin{abstract}
Background: Uveal melanoma (UM) is the most common primary ocular malignancy in adults in the Western world. UM with a mutation in SF3B1, a spliceosome gene, is characterized by three or more structural changes of chromosome 1, 6, 8, 9, or 11. Also UM without a mutation in SF3B1 harbors similar chromosomal aberrations. Since, in addition to SF3B1, mutations in U2AF1 and SRSF2 have also been observed in hematological malignancies, UM without a SF3B1 mutation-but with the characteristic chromosomal pattern-might harbor mutations in one of these genes. Methods: 42 UMs were selected based on their chromosomal profile and wildtype SF3B1 status. Sanger sequencing covering the U2AF1 (exon 2 and 7) hotspots and SRSF2 (exon 1 and 2) was performed on DNA extracted from tumor tissue. Data of three UM with an SRSF2 mutation was extracted from the The Cancer Genome Atlas (TCGA). Results: Heterozygous in-frame SRSF2 deletions affecting amino acids 92-100 were detected in two UMs ( $5 \%$ ) of 42 selected tumors and in three TGCA UM specimens. Both the UM with an SRSF2 mutation from our cohort and the UM samples from the TCGA showed more than four structural chromosomal aberrations including (partial) gain of chromosome 6 and 8 , although in two TCGA UMs monosomy 3 was observed. Conclusions: Whereas in myelodysplastic syndrome predominantly missense SRSF2 mutations are described, the observed SRSF2 mutations in UM are all in-frame deletions of 8-9 amino acids. This suggests that the R625 missense SF3B1 mutations and SRSF2 mutations in UM are different compared to the spliceosome gene mutations in hematological cancers, and probably target a different, as yet unknown, set of genes involved in uveal melanoma etiology.
\end{abstract}

Keywords: uveal melanoma; splicing; cancer; myelodysplastic syndrome

\section{Introduction}

Uveal melanoma (UM) is a primary malignant ocular tumor arising from melanocytes in the uvea which consist of iris, ciliary body, and choroid. Symptoms are present in the majority of patients with the most common presenting symptom being change in vision. Other presenting symptoms include photopsia and floaters [1]. Metastatic disease with predominantly metastasis to the liver, develops in almost half of all UM patients causing a poor prognosis [1,2]. Several prognostic factors are described with mutations in BAP1,SF3B1, and EIF1AX, with or without loss of chromosome 3, as important predictors of survival $[3,4]$. Tumors of uveal melanoma (UM) patients with somatic BAP1, SF3B1, or EIF1AX mutations show a distinct chromosomal copy number variation (CNV) pattern. Whereas EIF1AX ${ }^{\text {mut }}$ tumors in general lack gross anomalies, $B A P 1^{\text {mut }}$ tumors display monosomy 3 and isochromosome formation. $S F 3 B 1^{m u t}$ tumors are characterized by three or more structural 
variants, usually of chromosomes 1, 6, 8, 9, and 11 [5]. However, not all UMs with a typical SF3B1 ${ }^{\text {mut }}$ CNV harbor a mutation in the SF3B1 component of the spliceosome complex. As in myelodysplastic syndrome (MDS) and MDS-related diseases (such as chronic myelomonocytic leukemia and acute myloid leukemia) in which mutations in other genes of the spliceosome complex such as SRSF2 and $U 2 A F 1$ are described [6-10], mutations in SRSF2 and other spliceosome factors are also observed in UM [11]. Typical MDS-related mutations in SRSF2 involve codon 95 and are missense mutations resulting in an amino acid change (in $74 \%$ of patients with an SRSF2 mutation) or in-frame deletions starting at this codon (26\%) [8]. Missense mutations in U2AF1 in MDS are almost exclusively described in codon 34 (p.Ser34Phe and p.Ser34Tyr), 156 (Arg156His), or 157 (p.Gln157Arg and p.Gln157Pro) [7,12] . Therefore, mutation analysis of SRSF2 and U2AF1 covering these hotspots was performed on UM tumors with no SF3B1 mutation but with an SF3B1-like chromosomal CNV pattern.

\section{Results}

Heterozygous in-frame deletions starting at codon 92 of SRSF2 were identified in two of the selected 42 UM (p.(Tyr92_His99del); p.(Gly93_His100del)), (Figure 1). These mutations were mutually exclusive for BAP1, SF3B1, and EIF1AX but harbored a GNAQ p.(Gln209Leu) mutation (Table 1).
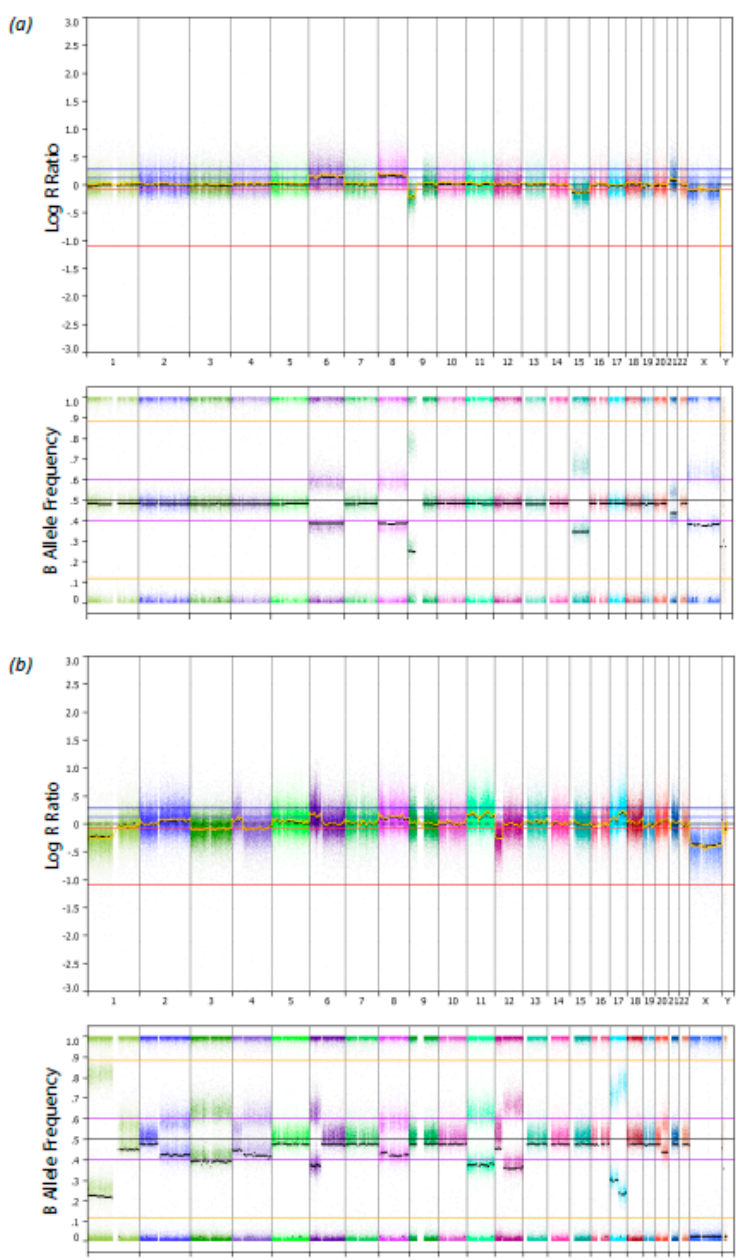

Figure 1. Single nucleotide polymorphism (SNP) array profile with the B-allele frequency from two uveal melanoma samples with an SRSF2 mutation. On the x-axes the chromosomes are displayed. (a) Uveal melanoma 1 (UM1). (b) Uveal melanoma 2 (UM2). 
Table 1. Overview of clinical characteristics, mutation status and copy number variation of uveal melanoma (UM) patients with an SRSF2 mutation.

\begin{tabular}{|c|c|c|}
\hline & UM 1 & UM 2 \\
\hline \multicolumn{3}{|l|}{ Clinical characteristics } \\
\hline Sex & Female & Male \\
\hline Age at diagnosis (years) & 63.0 & 57.3 \\
\hline Metastasis & No & No \\
\hline Disease free survival (months) & 76.8 & 128.8 \\
\hline \multicolumn{3}{|l|}{ Mutation status } \\
\hline SRSF2 & $\begin{array}{l}\text { Chr17(GRCh37):g.74732946_74732969del } \\
\text { c.274_297del:p.(Tyr92_His99del) }\end{array}$ & $\begin{array}{l}\text { Chr17(GRCh37):g.74732943_74732966del } \\
\text { c.277_300del:p.(Gly93_His100del) }\end{array}$ \\
\hline U2AF1 & Wildtype & Wildtype \\
\hline GNAQ & $\begin{array}{l}\text { Chr9(GRCh37):g.80409488T > A } \\
\text { c.626A > T:p.(Gln209Leu) }\end{array}$ & $\begin{array}{l}\text { Chr9(GRCh37):g.80409488T > A } \\
\text { c.626A > T:p.(Gln209Leu) }\end{array}$ \\
\hline GNA11 & Wildtype & Wildtype \\
\hline SF3B1 & Wildtype & Wildtype \\
\hline BAP1 & Wildtype & Wildtype \\
\hline EIF1AX & Wildtype & Wildtype \\
\hline Copy number variation & & \\
\hline (Partial) gain of chromosome & $6,8,21$ & $2 \mathrm{q}, 6 \mathrm{p}, 8,11,17,20 \mathrm{q}$ \\
\hline (Partial) loss of chromosome & $9 p, 15$ & $1 p, 3,4 q, 12 p$ \\
\hline
\end{tabular}

UM1 originates from the ciliary body and consists of mixed cell type with the presence of closed vascular loops. Largest tumor diameter was $19 \mathrm{~mm}$ with a prominence of $8 \mathrm{~mm}$. The other UM, UM2, arose from the choroid and consist of spindle cells. No closed vascular loops were present and there was no involvement of the ciliary body. The largest tumor diameter was $13 \mathrm{~mm}$ with a prominence of $5 \mathrm{~mm}$ with no extraocular extension.

Both UMs showed more than four chromosomal aberrations including gain of chromosome 6 and 8. The single nucleotide polymorphism (SNP) array profiles of these tumors are shown in Figure 2. Both patients did not develop metastatic disease and have a disease-free survival of 76.8 and 128.8 months, respectively. In none of the 42 samples a mutation in $U 2 A F 1$ was detected.
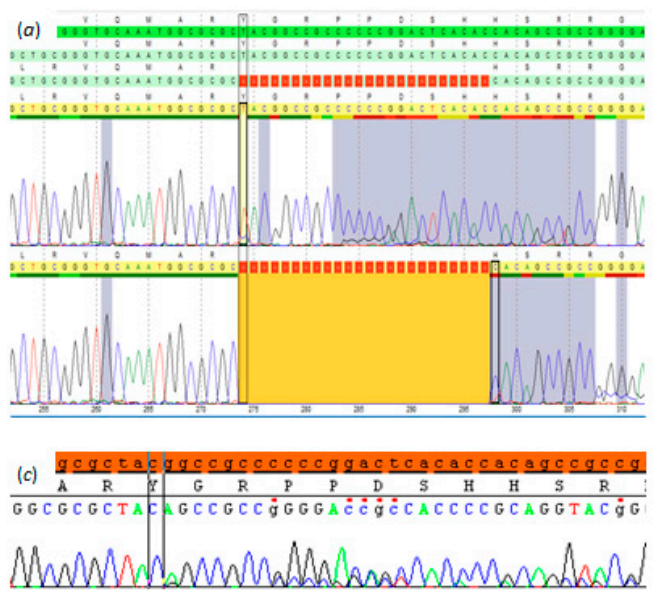
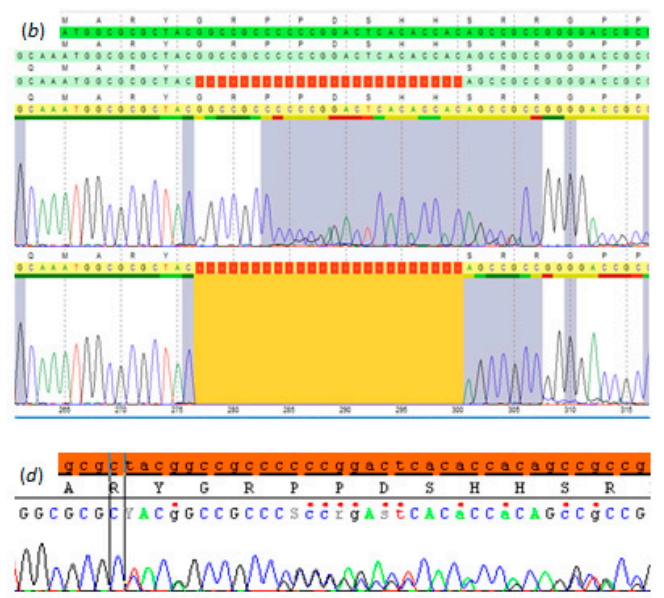

Figure 2. Mutations in the SRSF2 gene from two out of the 42 analyzed uveal melanoma patients. (a) and (c) Uveal melanoma sample (UM1) with a p.Y92_H99del displayed in SeqPilot V4.3.0 (JSI medical systems, Ettenheim, Germany) (a) and in SeqScape V3.0 (Thermo Fisher Scientific, Waltham, MA, USA) (c). (b,d) Uveal melanoma (UM2) with a p.G93_H100del displayed in SeqPilot V4.3.0 (b) and in SeqScape V3.0 (d).

Three previously described SRSF2 mutations were found in the data from the The Cancer Genome Atlas (TCGA) database [11]. CNV analysis showed loss of chromosome 3 in two UMs and gain of chromosome 8(q) in all three UMs. Gain of chromosome 1p was also present in two UMs and gain of chromosome 6 in one sample. Two UMs have a p.(Gln209Leu) mutation in GNAQ and one harbors a GNA11 mutation (p.(Gln209Leu)), (Table 2). No mutations in EIF1AX were detected, but one UM has BAP1 mutation (c.518A > G:p.(Tyr173Cys)). 
Table 2. Overview of mutations in uveal melanoma samples with an SRSF2 or U2AF1 mutation. ROMS = Rotterdam Ocular Melanoma Studygroup; TCGA = The Cancer Genome Atlas.

\begin{tabular}{|c|c|c|c|c|c|c|}
\hline & SRSF2 & U2AF1 & GNAQ & GNA11 & BAP1 & EIF1AX \\
\hline 1. ROMS & c.274_297del:p.(Tyr92_His99del) & Wildtype & c.626A > T:p.(Gln209Leu) & Wildtype & Wildtype & Wildtype \\
\hline 2. ROMS & c.277_300del:p.(Gly93_His100del) & Wild type & c.626A > T:p.(Gln209Leu) & Wildtype & Wildtype & Wildtype \\
\hline 3. TCGA & c.274_297del:p.(Tyr92_His99del) & Wildtype & c.626A > T:p.(Gln209Leu) & Wildtype & Wildtype & Wildtype \\
\hline 4. TCGA & c.274_300del:p.(Tyr92_His100del) & Wildtype & Wildtype & c.626A > T:p.(Gln209Leu) & Wildtype & Wildtype \\
\hline 5. TCGA & c.519_536del:p.(Ser174_Ser179del) & Wildtype & Wildtype & c.626A > T:p.(Gln209Leu) & c.518A > G:p.(Tyr173Cys) & Wildtype \\
\hline
\end{tabular}




\section{Discussion}

In this study we identified deletions in SRSF2 in two UM harboring an SF3B1 specific SNP array pattern albeit with no mutations of the SF3B1 hotspot regions. Studies have shown that in myelodysplastic syndrome (MDS) SRSF2 was mutated in $12-14 \%$ of the cases and mutations in U2AF1 occur in $15 \%$ of the MDS cases [7,8]. This is a higher frequency compared to UM, in which SRSF2 mutations are detected in less than $5 \%$ of the specimens and no U2AF1 mutations have been identified [11]. Three SRSF2 mutated UMs described in the literature are included in The Cancer Genome Atlas (TCGA, https://cancergenome.nih.gov/). Two out of these harbor similar deletions (Table 2) as we have identified in our cohort, and are mutually exclusive with BAP1 and EIF1AX, similar to our own observations. The third SRSF2 mutation from TCGA is a deletion of amino acid 174-179 and co-exists with a BAP1 mutation. Surprisingly, this tumor showed a BAP1 specific CNV profile, indicating that latter deletion of residues 174-179 has no or little pathogenic effect. However, other spliceosome gene mutations can underlie UM pathogenesis but might not display the same chromosomal anomalies as described in SF3B1 [5]. Furthermore, the low incidence of SRSF2 mutations in UM suggests that other genes of the splicing machinery, such as U2AF35 or ZRSR2, might be mutated. Mutations in other splicing genes than SF3B1 could be less frequently involved in the development of UM compared to MDS in which mutations in several splicing genes have been identified $[6,9,10]$.

Since Sanger sequencing was used for mutation analysis, we have focused on the hotspot regions of U2AF1 and SRSF2 that are described in UM and other diseases. More extensive research about mutations in all coding regions of these genes could increase the incidence.

Compared to SRSF2 mutations in MDS in which the vast majority are missense mutations [8], we observed a preference for in-frame deletions in UM. Also, for SF3B1 in UM residue R625 is most commonly mutated residue, whereas in other tumors predominantly the K700 residue of SF3B1 is affected $[6,11]$. Thus, although the same gene is involved, mutations occur on different residues in distinct diseases. Furthermore, studying the RNA expression of SRSF2 mutated UM from TGCA, we did not observe the same splicing effect as observed in SF3B1 mutated UM. These findings suggest that SF3B1 mutations compared to mutations in SRSF2 have, despite a similar chromosomal pattern, a different effect on splicing.

Since we observed SRSF2 mutations in only two patients the clinical impact of this mutation remains unclear. However, both patients with an SRSF2 mutation in our cohort did not develop metastasis within 6 and 10 years, neither did the patients from TCGA. In chronic myelomonocytic leukemia no difference in overall survival was observed, and not in MDS when corrected for age $[8,10]$. Future studies are needed to evaluate the role of other splicing genes than SF3B1 in UM.

\section{Materials and Methods}

Patients with an SF3B1-like chromosomal pattern were selected from the Rotterdam Ocular Melanoma Study group (ROMS) database. These UM patients underwent enucleation or biopsy of the tumor in the Erasmus Medical Center (Rotterdam, The Netherlands) or The Rotterdam Eye Hospital (Rotterdam, the Netherlands) between 1993 and 2017. Informed consent from all patients was obtained before collecting the tumor material. This study was performed according to the tenets of the Declaration of Helsinki and approved by the local ethics committee (MEC-2009-375, 12th November 2009).

DNA was isolated from fresh tumor tissue using the QIAamp DNA Mini Kit (Qiagen, Hilden, Germany) and concentrations measured using the Quant-iT PicoGreen dsDNA Assay Kit (Thermo Fisher Scientific, Waltham, MA, USA). Two hundred nanograms of DNA input was used for SNP-array analysis using an Illumina Human SNP array platform (Illumina, San Diego, CA, USA). Copy number analysis was performed using Nexus Copy Number 8.0 (BioDiscovery, El Segundo, CA, USA). Moreover, karyotyping was used for $\mathrm{CNV}$ analysis when available. Patients were selected from the cohort described previously [5]. 
In general, an SF3B1-like chromosomal pattern is defined as a combination of three structural variations in SNP array analysis of the tumor (usually this includes either partial gain of chromosome $8 q$ or $9 q$ or partial loss of chromosome 1p or 11q) [5]. In addition, UM with gain of chromosome $6 p$ or loss of $6 \mathrm{q}$ in addition to one or two other anomalies were also included since these anomalies are also specific for SF3B1 mutated tumors, whereas this is not seen in EIF1AX or BAP1 mutated UM. Moreover, solely gain of chromosome $6 \mathrm{p}$ was only included when the tumor did not harbor an EIF1AX mutation, because gain of chromosome $6 \mathrm{p}$ is only representative for SF3B1 and EIF1AX mutated UM.

The two coding exons of the SRSF2 gene were sequenced using Sanger sequencing with primers for these regions (pxlence, Dendermonde, Belgium). The mutation hotspots in U2AF1 were sequenced with primers covering codon 34,156, and 157. Sanger sequence results were visualized with SeqScapeSoftware V3.0 (Thermo Fisher Scientific, Waltham, MA, USA) and SeqPilot V4.3.0 (JSI medical systems $\mathrm{GmbH}$, Ettenheim, Germany).

Mutation analysis of GNAQ, GNA11, SF3B1, BAP1, and EIF1AX was performed previously using Sanger sequencing and next-generation sequencing using the ION Torrent platform (Life Technologies, Carlsbad, CA, USA) [5,13]. A BAP1 mutation was defined as a mutation in the BAP1 gene or lack of nuclear BAP1 expression (performed as described previously [14]).

The UM cohort from the National Insitute of Health TCGA server $(n=80)$ was used for mutation analysis of SRSF2 and U2AF1 using Integrative Genomics Viewer (Version 2.3.68 (97) (Broad Institute, Cambridge, MA, USA). If a mutation in one of these genes was identified, copy number analysis was performed on the segmented SNP array data using Nexus Copy Number 8.0 (BioDiscovery, El Segundo, CA, USA).

\section{Conclusions}

UMs harbor chromosomal aberrations correlated with their mutation status [5]. Mutations in SF3B1 and SRSF2, genes that are both involved in splicing, occur not only in UM but are described in MDS and MDS related diseases as well [8-11,15]. However, the mutation type in these genes are different in both diseases. In UM, SF3B1 is almost exclusively a missense mutation at residue 625 whereas in other diseases residue 700 is mutated [6,11]. In SRSF2, a different type of mutation is also observed in UM compared to MDS and MDS related diseases, but the same region is involved. We identified in-frame deletions of SRSF2 in UM in the same genetic region, whereas most mutations in the same gene in MDS are missense mutations [8]. Therefore, we conclude that there might be a preference for in-frame deletions in SRSF2 in UM when this gene is involved. We did not observe any mutation in U2AF1 in our selected cohort, and the incidence of mutations of SRSF2 is low. Although we have a selected cohort which might influence the incidence, this is in line with previous studies, in which no or few mutations in these genes are found in UM patients $[11,16,17]$. The clinical relevance of CNV pattern and the relation to spliceosome mutations remains unclear. More research is needed to evaluate the significance of these findings.

Author Contributions: Conceptualization, A.d.K., E.K.; methodology, A.d.K., E.K., N.M.v.P.; software, T.B.; validation, N.M.v.P., W.D.; formal analysis, E.K., A.d.K., N.M.v.P.; investigation, N.M.v.P., W.D.; resources, E.K., D.P., K.N.S., J.V., N.M.v.P., W.D.; data curation, N.M.v.P., E.K., A.d.K.; writing—original draft preparation, N.M.v.P.; writing-review and editing, J.V., W.D., K.N.S., T.B., D.P., E.K., A.d.K., visualization, N.M.v.P., T.B.; supervision, A.d.K., E.K.; project administration, E.K., A.d.K.; funding acquisition, A.d.K., E.K.

Funding: This research was funded by the Dutch Cancer Society, grant number EMCR 2014-6905.

Acknowledgments: The authors would like to thank Merel van Dijk and Shirin Mostert for their help in patient selection and mutation analysis.

Conflicts of Interest: The authors declare no conflict of interest. The funders had no role in the design of the study; in the collection, analyses, or interpretation of data; in the writing of the manuscript, or in the decision to publish the results. 


\section{References}

1. Delgado-Ramos, G.M.; Thomas, F.; VanderWalde, A.; King, B.; Wilson, M.; Pallera, A.M. Risk factors, clinical outcomes, and natural history of uveal melanoma: A single-institution analysis. Med. Oncol. 2019, 36, 17. [CrossRef] [PubMed]

2. Vichitvejpaisal, P.; Dalvin, L.A.; Mazloumi, M.; Ewens, K.G.; Ganguly, A.; Shields, C.L. Genetic Analysis of Uveal Melanoma in 658 Patients using The Cancer Genome Atlas (TCGA) Classification of Uveal Melanoma as A, B, C \& D. Ophthalmology 2019, in press.

3. Yavuzyigitoglu, S.; Koopmans, A.E.; Verdijk, R.M.; Vaarwater, J.; Eussen, B.; Van Bodegom, A.; Paridaens, D.; Kiliç, E.; de Klein, A. Rotterdam Ocular Melanoma Study Group. Uveal Melanomas with SF3B1 Mutations: A Distinct Subclass Associated with Late-Onset Metastases. Ophthalmology 2016, 123, 1118-1128. [CrossRef] [PubMed]

4. Ewens, K.G.; Kanetsky, P.A.; Richards-Yutz, J.; Purrazzella, J.; Shields, C.L.; Ganguly, T.; Ganguly, A. Chromosome 3 status combined with BAP1 and EIF1AX mutation profiles are associated with metastasis in uveal melanoma. Investig. Ophthalmol. Vis. Sci. 2014, 55, 5160-5167. [CrossRef] [PubMed]

5. Yavuzyigitoglu, S.; Drabarek, W.; Smit, K.N.; van Poppelen, N.; Koopmans, A.E.; Vaarwater, J.; Brands, T.; Eussen, B.; Dubbink, H.J.; van Riet, J.; et al. Correlation of Gene Mutation Status with Copy Number Profile in Uveal Melanoma. Ophthalmology 2017, 124, 573-575. [CrossRef] [PubMed]

6. Yoshida, K.; Sanada, M.; Shiraishi, Y.; Nowak, D.; Nagata, Y.; Yamamoto, R.; Sato, Y.; Sato-Otsubo, A.; Kon, A.; Nagasaki, M.; et al. Frequent pathway mutations of splicing machinery in myelodysplasia. Nature 2011, 478, 64-69. [CrossRef] [PubMed]

7. Tefferi, A.; Mudireddy, M.; Finke, C.M.; Nicolosi, M.; Lasho, T.L.; Hanson, C.A.; Patnaik, M.M.; Pardanani, A.; Gangat, N. U2AF1 mutation variants in myelodysplastic syndromes and their clinical correlates. Am. J. Hematol. 2018, 93, E146-E148. [CrossRef] [PubMed]

8. Wu, S.J.; Kuo, Y.Y.; Hou, H.A.; Li, L.Y.; Tseng, M.H.; Huang, C.F.; Lee, F.Y.; Liu, M.C.; Liu, C.W.; Lin, C.T.; et al. The clinical implication of SRSF2 mutation in patients with myelodysplastic syndrome and its stability during disease evolution. Blood 2012, 120, 3106-3111. [CrossRef] [PubMed]

9. Je, E.M.; Yoo, N.J.; Kim, Y.J.; Kim, M.S.; Lee, S.H. Mutational analysis of splicing machinery genes SF3B1, U2AF1 and SRSF2 in myelodysplasia and other common tumors. Int. J. Cancer 2013, 133, 260-265. [CrossRef] [PubMed]

10. Meggendorfer, M.; Roller, A.; Haferlach, T.; Eder, C.; Dicker, F.; Grossmann, V.; Kohlmann, A.; Alpermann, T.; Yoshida, K.; Ogawa, S.; et al. SRSF2 mutations in 275 cases with chronic myelomonocytic leukemia (CMML). Blood 2012, 120, 3080-3088. [CrossRef] [PubMed]

11. Field, M.G.; Durante, M.A.; Anbunathan, H.; Cai, L.Z.; Decatur, C.L.; Bowcock, A.M.; Kurtenbach, S.; Harbour, J.W. Punctuated evolution of canonical genomic aberrations in uveal melanoma. Nat. Commun. 2018, 9, 116. [CrossRef] [PubMed]

12. Graubert, T.A.; Shen, D.; Ding, L.; Okeyo-Owuor, T.; Lunn, C.L.; Shao, J.; Krysiak, K.; Harris, C.C.; Koboldt, D.C.; Larson, D.E.; et al. Recurrent mutations in the U2AF1 splicing factor in myelodysplastic syndromes. Nat. Genet. 2011, 44, 53-57. [CrossRef] [PubMed]

13. Smit, K.N.; Van Poppelen, N.M.; Vaarwater, J.; Verdijk, R.; van Marion, R.; Kalirai, H.; Coupland, S.E.; Thornton, S.; Farquhar, N.; Dubbink, H.J.; et al. Combined mutation and copy-number variation detection by targeted next-generation sequencing in uveal melanoma. Mod. Pathol. 2018, 31, 763-771. [CrossRef] [PubMed]

14. Koopmans, A.E.; Verdijk, R.M.; Brouwer, R.W.; Van Den Bosch, T.P.; Van Den Berg, M.M.; Vaarwater, J.; Kockx, C.E.; Paridaens, D.; Naus, N.C.; Nellist, M.; et al. Clinical significance of immunohistochemistry for detection of BAP1 mutations in uveal melanoma. Mod. Pathol. 2014, 27, 1321-1330. [CrossRef] [PubMed]

15. Yang, J.; Yao, D.M.; Ma, J.C.; Yang, L.; Guo, H.; Wen, X.M.; Xiao, G.F.; Qian, Z.; Lin, J.; Qian, J. The prognostic implication of SRSF2 mutations in Chinese patients with acute myeloid leukemia. Tumour Biol. 2016, 37, 10107-10114. [CrossRef] [PubMed] 
16. Afshar, A.R.; Damato, B.E.; Stewart, J.M.; Zablotska, L.B.; Roy, R.; Olshen, A.B.; Joseph, N.M.; Bastian, B.C. Next-Generation Sequencing of Uveal Melanoma for Detection of Genetic Alterations Predicting Metastasis. Transl. Vis. Sci. Technol. 2019, 8, 18. [CrossRef] [PubMed]

17. Robertson, A.G.; Shih, J.; Yau, C.; Gibb, E.A.; Oba, J.; Mungall, K.L.; Hess, J.M.; Uzunangelov, V.; Walter, V.; Danilova, L.; et al. Integrative Analysis Identifies Four Molecular and Clinical Subsets in Uveal Melanoma. Cancer Cell 2017, 32, 204-220. [CrossRef] [PubMed]

C 2019 by the authors. Licensee MDPI, Basel, Switzerland. This article is an open access article distributed under the terms and conditions of the Creative Commons Attribution (CC BY) license (http://creativecommons.org/licenses/by/4.0/). 